\title{
Variant of takotsubo cardiomyopathy associated with sepsis and respiratory failure in an elderly female*
}

\author{
Punnaiah Marella $^{1 \#}$, Shantipriya Siripurapu ${ }^{1}$, Hassan Hussein ${ }^{2}$, Rajeev Garg ${ }^{3,4,5}$ \\ ${ }^{1}$ Department of Internal Medicine, Banner Estrella Medical Center, Phoenix, USA \\ ${ }^{2}$ Department of Internal Medicine, West Valley Hospital, Goodyear, USA \\ ${ }^{3}$ Department of Cardiology, Banner Estrella Medical Center, Phoenix, USA \\ ${ }^{4}$ Banner Boswell Medical Center, Sun City, USA \\ ${ }^{5}$ West Valley Hospital, Goodyear, USA \\ Email: "chowdarymp@yahoo.com
}

Received 1 November 2012; revised 1 December 2012; accepted 13 December 2012

\begin{abstract}
Stress induced cardiomyopathy/Takotsubo cardiomyopathy (TSO CMO) has been widely reported. It is characterized by apical hypokinesis or akinesis. Variants of this called as inverted/reverse cardiomyopathies have been reported and are characterized by basal hypokinesis/akinesis and hypercontractility of apex. These are more common in younger population. We present an elderly female who had a variant cardiomyopathy in association with sepsis and respiratory failure and this has been rarely reported. An 84-yearold female presented with cough, dyspnea and fevers. She was treated for pneumonia but her respiratory failure worsened and she suffered a non ST segment elevation myocardial infarction. Cardiac catheterization revealed normal coronaries but ejection fraction was low at $\mathbf{2 5 \%}$ with basal hypokinesis and a hyperkinetic apex. She improved with diuresis and medical management of a variant of stress induced cardiomyopathy. Stress induced cardiomyopathies and its variants are reversible conditions and improve with conservative management. These entities should be kept in mind during investigation of any acute myocardial infarction.
\end{abstract}

Keywords: Takotsubo Cardiomyopathy; Inverted Cardiomyopathy; Stress Induced Cardiomyopathy

\section{INTRODUCTION}

Takotsubo cardiomyopathy (TSO CMO) is reversible left ventricular apical ballooning without any significant coronary artery disease [1]. Multiple variants characterized

\footnotetext{
*Institution at which work was done: Banner Boswell Medical Center, 13203 N $103^{\text {rd }} A v$, \#H5, Sun City, AZ 85351.

Financial disclosures and conflicts of interest: None.

"Corresponding author.
}

by midventricular and basal akinesis with a hypercontractile apex have been reported. High index of suspicion is needed for diagnosis, especially in patients presenting with acute myocardial infarction picture but without risk factors for coronary artery disease. Hence, thrombolytics should not be given as there is no major coronary artery disease. Diagnosis is usually made by an echocardiogram and a cardiac catheterization is warranted to make sure there is no coronary artery disease. Patients recover quickly with the conventional treatment of cardiomyopathy and ejection fraction normalizes in a few weeks [2]. We present this case of an 84-year-old female who had basal hypokinesis and was in sepsis and respiratory failure.

\section{CASE REPORT}

An 84-year-old woman presented with dyspnea of 1week duration. She had hypothyroidism, chronic obstructive pulmonary disease and prior atypical mycobacterium avium intracellulare complex (MAC) infection of lungs treated 10 years ago. She had associated fever and cough. Physical exam was consistant with bibasal crepitations in lungs. She did not have a raised jugular venous distension or pedal edema or a cardiac murmur. She had a temperature of 38 degrees Celcius, otherwise vital signs were unremarkable. Complete blood counts with differential and comprehensive metabolic panel were normal. Chest X-ray showed bilateral infiltrates. Electrocardiogram did not reveal any ischemic patterns. Computerised axial tomography scan of thorax showed diffuse bronchiectasis and bronchiolitis with infiltrates. Pulmonary function tests showed obstructive disease with severe restriction in diffusion. Despite antibiotics for pneumonia, her respiratory status worsened and she was intubated. She was started on steroids and underwent a bronchoscopy. Troponins peaked at $0.91 \mathrm{ng} / \mathrm{mL}$ and trended down to 0.11 . Pro-brain natriuretic peptide was $11,959 \mathrm{pg} / \mathrm{mL}$. 
Echocardiogram showed an ejection fraction (EF) of $45 \%$, diastolic dysfunction, moderate mitral regurgitation, and moderate tricuspid regurgitation. She was extubated 2 days later. Blood cultures and bronchoalveolar lavage cultures did not show growth of any organisms. Sputum acid fast bacillus (AFB) stain was positive for MAC. Respiratory status worsened again and she required noninvasive ventilation temporarily. She was started on diuretics. Left and right heart catheterization showed normal coronaries, an EF of $25 \%$ - 30\% with apical hyperkinesis and basal hypokinesis (Figures 1 and 2). Diuresis was increased for the newly diagnosed nonischemic cardiomyopathy. We started ethambutol and azithromycin for

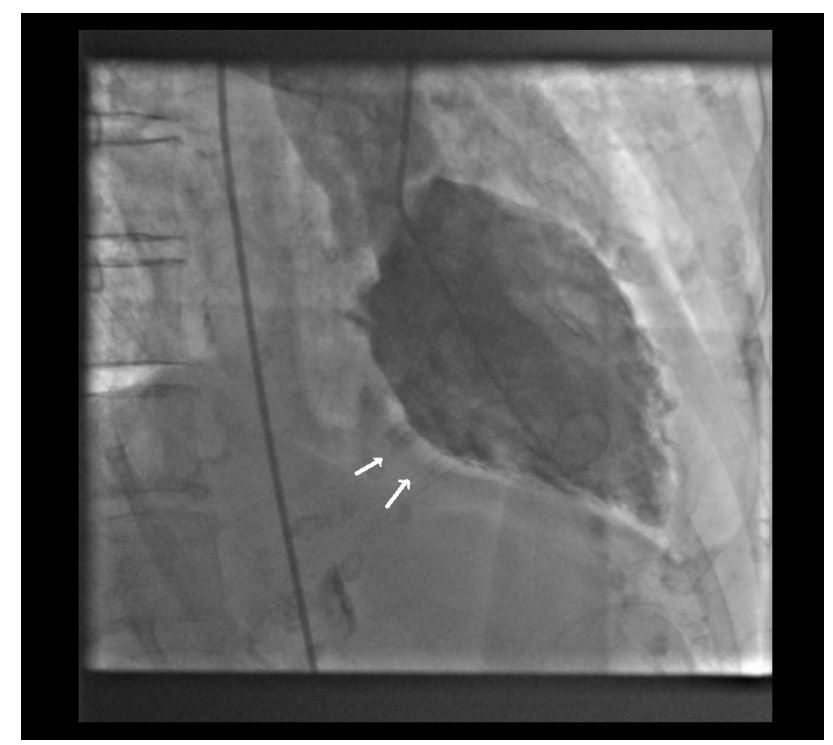

Figure 1. Left ventriculogram in systole showing basal hypokinetic areas seen as ballooning (Arrow).

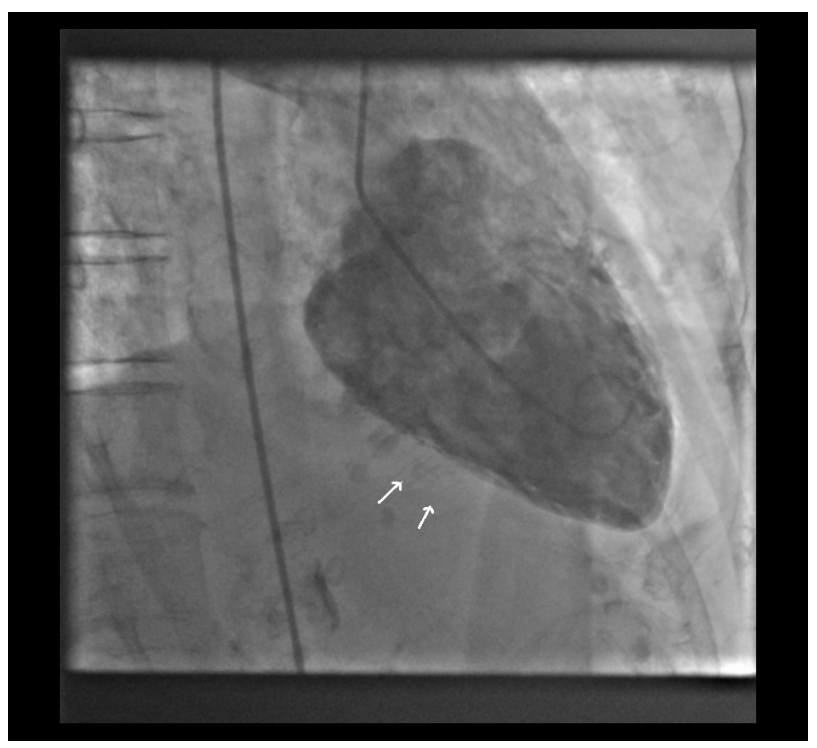

Figure 2. Left ventriculogram in diastole showing hypokinetic areas in diastole (Arrow). recurrence of MAC pneumonia. After a brief hospital course, she was discharged home in a stable condition on tapering steroids, aspirin, betablocker, angiotensin converting enzyme inhibitor, lasix, azithromycin and ethambutol. A follow-up echocardiogram 2 months later showed a normal EF of $55 \%$.

\section{DISCUSSION}

Stress induced cardiomyopathy/Takotsubo cardiomyopathy (TSO CMO)/transient left ventricular (LV) apical ballooning/broken heart syndrome was first reported in Japan [1]. The name came from the similarity of the ventriculogram to the instrument used in Japan to trap an Octopus. The condition is characterized by transient LV apical wall motion abnormality. This classic type mimics acute coronary syndrome with electrocardiogram changes and troponin leak but there is no significant coronary artery disease on angiography [2]. $80 \%$ of patients are usually post-menopausal women [3]. An associated medical illness or severe psychological stress is a common provocating factor [3]. Presentation is same as any acute myocardial infarction and the most common initial symptom is chest pain. Electrocardiographic (EKG) abnormalities can vary. An anterior ST elevation type of presentation is seen in approximately $80 \%$ of patients [4]. Other EKG presentations could be in form of ST segment depression, abnormal Q waves, prolonged QT interval, $\mathrm{T}$ wave inversions and there are no reciprocal changes in this entity [4]. Multiple etiologies for these cardiomyopathies have been hypothesized including catecholamine excess, neurogenic stunning due to emotional or physical stress, microvascular dysfunction and multivessel coronary spasm [5]. Diagnosis is supported by an echocardiogram with confirmation of segmental wall motion abnormalities. A cardiac catheterization is warranted to rule out coronary artery disease. It is unclear why the cardiac apex is specifically involved. Possible explanations could be lack of three layered myocardial structure, apex being in border zone of perfusion of major coronary arteries and loss of elasticity after expansion [3].

There are variant types of TSO CMO, also called as midventricular ballooning syndrome/inverted or reverse TSO CMO and account for $40 \%$ of cases [6]. These variant types are characterized by basal and midportion akinesis and hyperdynamic apex. These variations in regional wall motion abnormalities could be due to location of cardiac adrenergic receptors, degree of excess sympathetic activity and variation of sympathetic stimulation susceptibility between individuals [2]. Differences in heart rate dynamics, sympathetic activation and reflex autonomic regulation may explain some of the differences between the typical and atypical presentations [7]. Variants have been associated with severe stressful conditions like 
cerebrovascular accident, pheochromocytoma, and pancreatitis, only to name a few. Catecholamine excess was a likely phenomenon in most of these cases. Patients with the variant types were shown to be significantly younger than the classic pattern [8]. It was hypothesized that in young people there is more density of adrenergic receptor in base and hence could be causing basal hypokinesis [9]. Significant relation with hypertension and pheochromocytoma is to be excluded. Diagnosis of any of these cardiomyopathies is usually made by echocardiogram and cardiac catheterization. Even myocardial biopsies failed to add further information to the exact pathophysiology of these conditions and it is unlikely for myocardial inflammation to affect only some parts of the heart [10]. After the initial injury, the overall course of the disease tends to be good and overall prognosis is usually good [10]. Patients recover quickly and treatment is same as of any cardiomyopathy and EF normalizes in a few weeks' time.

\section{CONCLUSION}

Based on the highly propagated theory of catecholamine excess in these cardiomyopathies, variant cardiomyopathies are more common in younger population as they have higher density of adrenergic receptor in base of heart. Contrary to this theory our patient was an elderly patient and had predominant basal hypokinesis with hyperkinetic apex. An association of this variant TSO CMO with sepsis and respiratory failure has rarely been reported. Stress induced cardiomyopathies and its variants should always be considered in any patient presenting with myocardial infarction. Thrombolytics should not be used and a timely echocardiogram and cardiac catheterization are helpful.

\section{ACKNOWLEDGEMENTS}

Our sincere thanks to Joe Huizar, senior manager of medical imaging in Banner Estrella Medical center, for his help and technical support.

\section{REFERENCES}

[1] Dote, K., Sato, H., Tateishi, H., Uchida, T. and Ishihara, M. (1991) Myocardial stunning due to simultaneous multivessel coronary spasms: A review of 5 cases. Journal of Cardiology, 1, 203-214.
[2] Yasu, T., Tone, K., Kubo, N. and Saito, M. (2006) Transient mid-ventricular ballooning cardiomyopathy: A new entity of takotsubo cardiomyopathy. International Journal of Cardiology, 110, 100-101. doi:10.1016/j.ijcard.2005.05.060

[3] Nanda, S., Pamula, J., Bhatt, S.P., Chan, I., Turki, M.A. and Dale, T.H. (2007) Takotsubo cardiomyopathy-A new variant and widening disease spectrum. International Journal of Cardiology, 120, e34-e36. doi:10.1016/j.ijcard.2007.04.067

[4] Gianni, M., Dentali, F., Grandi, A.M., Sumner, G., Hiralal, R. and Lonn, E. (2006) Apical ballooning syndrome or takotsubo cardiomyopathy: A systematic review. European Heart Journal, 27, 1523-1529. doi:10.1093/eurheartj/ehl032

[5] Sasaki, O., Nishioka, T., Akima, T., Tabata, H., Okamoto, Y., Akanuma, M., et al. (2006) Association of takotsubo cardiomyopathy and long QT syndrome. Circulation Journal, 70, 1220-1222. doi:10.1253/circj.70.1220

[6] Kurowski, V., Kaiser, A., von Hof, K., Killermann, D.P., Mayer, B., Hartmann, F., et al. (2007) Apical and midventricular transient left ventricular dysfunction syndrome (tako-tsubo cardiomyopathy): Frequency, mechanisms, and prognosis. Chest, 132, 809-816. doi:10.1378/chest.07-0608

[7] Bonnemeier, H., Demming, T., Weidtmann, B., Ortak, J., Burgdorf, C., Reppel, M., et al. (2010) Differential heart rate dynamics in transient left ventricular apical and midventricular ballooning. Heart Rhythm, 7, 1825-1832. doi:10.1016/j.hrthm.2010.08.025

[8] Mansencal, N., Abbou, N., N’Guetta, R., Pilliere, R., El Mahmoud, R. and Dubourg, O. (20110) Apical-sparing variant of tako-tsubo cardiomyopathy: Prevalence and characteristics. Archives of Cardiovascular Diseases, 103, 75-79.

[9] Ramaraj, R. and Movahed, M.R. (2010) Reverse or inverted takotsubo cardiomyopathy (reverse left ventricular apical ballooning syndrome) presents at a younger age compared with the mid or apical variant and is always associated with triggering stress. Congestive Heart Failure, 16, 284-286. doi:10.1111/j.1751-7133.2010.00188.x

[10] Fazio, G., Barbaro, G., Sutera, L., Guttilla, D., Pizzuto, C., Azzarelli, S., et al. (2008) Clinical findings of takotsubo cardiomyopathy: Results from a multicenter international study. Journal of Cardiovascular Medicine (Hagerstown), 9, 239-244. doi:10.2459/JCM.0b013e328216276d 